\title{
DESENVOLVIMENTO DE MUDAS DE CITROS CULTIVADAS EM VASO EM RESPOSTA À ADUBAÇÃO NPK ${ }^{1}$
}

\author{
Alberto Carlos de Campos Bernardi2,5*; Quirino Augusto de Camargo Carmello³; Sérgio Alves \\ de Carvalho ${ }^{4}$ \\ ${ }^{2}$ Embrapa Solos - R. Jardim Botânico, 1024 - CEP: 22460-000 - Rio de Janeiro, RJ. \\ ${ }^{3}$ Depto. de Solos e Nutrição de Plantas - USP/ESALQ, C.P. 9 - CEP: 13418-900 - Piracicaba, SP. \\ ${ }^{4}$ Centro de Citricultura Sylvio Moreira - IAC, C.P. 4 - CEP: 13490-970 - Cordeirópolis, SP. \\ ${ }^{5}$ Bolsista CNPq. \\ *Autor correspondente <alberto@cnps.embrapa.br>
}

\begin{abstract}
RESUMO: O objetivo deste trabalho foi avaliar o efeito da adubação NPK sobre o desenvolvimento de mudas de laranjeira 'Valência' (Citrus sinensis) sobre o porta-enxerto de limoeiro 'Cravo' (C. limonia) em vasos com substrato de casca de Pinus, vermiculita e perlita. Utilizou-se um esquema de fatorial fracionário $(1 / 5) 5^{3}$ com um delineamento em blocos ao acaso. Os tratamentos consistiram em 5 doses (em g por planta) de N: 1,25; 6,25; 11,25; 16,25; 21,$25 ; 5$ doses de K: 0,$42 ; 3,75 ; 6,22 ; 9,34 ; 12,45$; e 5 doses de P: 0,$19 ; 0,89 ; 1,59 ; 2,29 ; 2,99$. O N e o K foram fornecidos semanalmente, sendo $37,5 \%$ da dose para o porta-enxerto. O P foi fornecido totalmente no plantio. Avaliaram-se a produção de material seco das folhas, raízes, caule, parte aérea e total; área foliar, relação raízes/ parte aérea; altura de plantas; volume radicular, relação área foliar/material seco para os porta-enxertos e as mudas, e o diâmetro do caule para os porta-enxertos. Funções de resposta foram ajustadas e os níveis de nutrientes foram calculados. Os resultados mostraram que as doses de fertilizantes que levaram às melhores respostas para produção de mudas de laranjeira 'Valência'/'Cravo' sobre o porta-enxertos de limoeiro 'Cravo' foram: N, 9,85; P, 2,86; K, 7,99 g por plantas.
\end{abstract}

Palavras-chave: Citrus sinensis, Citrus limonia, propagação, superfície de resposta, adubação

\section{DEVELOPMENT OF CITRUS NURSERY TREES GROWN IN POTS IN RESPONSE TO NPK FERTILIZATION}

\begin{abstract}
The objective of this research was to evaluate the NPK fertilizer effect on 'Valencia' sweet orange nursery tree (Citrus sinensis) development, budded on Rangpur lime rootstocks (C. limonia) in a protected environment using containers with Pinus bark, vermiculite and perlite substrates. The experiment consisted of a complete $(1 / 5) 5^{3}$ factorial randomized block design. Treatments comprised five concentrations in $\mathrm{g}$ per plant of $\mathrm{N}: 1.25 ; 6.25 ; 11.25 ; 16.25 ; 21.25$; five concnetrations of $\mathrm{K}: 0.42 ; 3.75 ; 6.22 ; 9.34 ; 12.45$; and five concentration of $\mathrm{P}: 0.19 ; 0.89 ; 1.59 ; 2.29 ; 2.99$. Nitrogen and $\mathrm{K}$ were applieded weekly, and $37.5 \%$ of the total fertilization was applied to rootstocks. All $\mathrm{P}$ was supplied at planting. Leaf, root, stem, shoot and total dry matter production, shoot/root ratio, leaf area, plant height, root system volume; leaf area dry matter ratio stem diameter were evaluated. Response functions were adjusted and nutrient rates for maximum yield are presented. Results showed that fertilizer level that lead to the best 'Valencia' sweet orange on Rangpur lime nursery tree response was (g per plant): N, 9.85; P, 2.86; K, 7.99.

Key words: Citrus sinensis, Citrus limonia, propagation, response function, fertilizer
\end{abstract}

\section{INTRODUÇÃO}

A produção de mudas de citros em ambiente protegido é uma alternativa ao sistema tradicional de produção em viveiros a campo. O sistema propõe a produção sob casa-de-vegetação onde os porta-enxertos são cultivados em tubetes com diferentes substratos, com posterior transplantio para vasos maiores para realização da enxertia e formação da muda (Carvalho, 1998). O objetivo é melhorar as condições fitossanitárias, promover um crescimento mais intenso e padronizar o processo de formação das mudas. No entanto, nesse sistema de produção ocorre um grande crescimento das plantas em curto espaço de tempo e em espaço reduzido para o desenvolvimento do sistema radicular (Carvalho, 1994). Portanto, o fornecimento de nutrientes em doses adequadas e balanceadas é necessário para estimular o crescimento máximo e para que perdas por lixiviação sejam evitadas.

A produção de mudas cítricas em vasos e em ambiente protegido vem sendo utilizada na Flórida (EUA) desde 1977, mas ainda existe grande variação nas doses e freqüências de aplicação de fertilizantes. As adubações nitrogenadas são aplicadas com base em dosagens utilizadas em viveiros no campo devido à ausência de recomendações na literatura (Castle \& Fergunson, 1982 e Maust \& Williamson, 1994). 
No Brasil, os estudos realizados com 0 fornecimento de fertilizantes contendo $\mathrm{N}, \mathrm{P}$ e K ao limoeiro 'Cravo' até o ponto de repicagem ou transplantio para vasos definitivos têm-se mostrado benéficos, pois obtiveram-se redução do tempo de formação e aumento da produção de material seco das plantas (Carvalho \& Souza, 1988; Carvalho, 1994; DeCarlos Neto et al., 1994). Após o transplante, durante a fase inicial de crescimento do porta-enxerto, existem respostas ao $P$ no substrato muito superiores às normalmente observadas no solo, registrando-se casos de respostas positivas até $2,2 \mathrm{~kg} \mathrm{~m}^{-3}$ de P (Resende et al., 1995).

Pesquisas têm mostrado que os nutrientes interferem no crescimento das plantas, mas é necessário estabelecer as doses adequadas para tornar a produção economicamente viável e maximizar o crescimento, pois os desbalanços nutricionais podem acarretar prejuízos à muda, alterando sua morfologia. Existem estudos mostrando que doses elevadas de $\mathrm{N}$ podem ser prejudiciais ao desenvolvimento radicular (Ford et al., 1957; Smith, 1965; Witt, 1997) e alteram a relação entre as raízes e a parte aérea (Marschner, 1995).

Outro fator que deve ser considerado é que, na cultura dos citros, o crescimento das raízes e da parte aérea segue um ritmo alternado. Bevington \& Castle (1985) determinaram que nas plantas jovens de citros, mesmo quando a temperatura e a umidade do solo não são fatores limitantes, o crescimento das raízes é cíclico, alternando-se com o crescimento da parte aérea. Observaram em plantas de laranjeira 'Valência' sobre limoeiro 'Rugoso' e citrange 'Carrizo', que a redução no crescimento do sistema radicular ocorreu na mesma proporção do aumento da taxa de crescimento da parte aérea. Essa situação fica mais evidenciada após a enxertia, quando faz-se o forçamento da borbulha do enxerto e sua brotação de folhas restitui a parte área da planta. Nesse momento, a variedade copa enxertada tem prioridade e seu crescimento ocorre em detrimento do crescimento das raízes (Castle, 1978). Isso ocorre, pois as raízes e a parte aérea competem por água, nutrientes e carbono (Castle, 1978; Syvertsen \& Lloyd, 1994) e pelas reservas de nitrogênio (Legaz et al., 1995). Além da água e dos nutrientes, os fitorreguladores (citocininas e ácido abscísico) e outros metabólitos também exercem um papel importante na relação raízes / parte aérea dos citros (Syvertsen \& Lloyd, 1994; Witt, 1997).

O objetivo deste trabalho foi avaliar o efeito da adubação com NPK sobre o crescimento e a produção de matéria seca do porta-enxerto limoeiro 'Cravo' e das mudas de laranjeira 'Valência'/'Cravo' produzidas em vasos sob ambiente protegido.

\section{MATERIAL E MÉTODOS}

O experimento foi conduzido em viveiro demonstrativo do sistema de produção de mudas certificadas de citros, no Centro de Citricultura "Sylvio Moreira", do Instituto Agronômico - IAC, em Cordeirópolis-
SP. O viveiro é coberto por filme plástico transparente e com telado contra afídeos nas laterais. As bancadas para a produção de mudas são elevadas em 0,3 m do solo (Carvalho \& Laranjeira, 1994). Utilizou-se o substrato comercial Rendimax, composto de casca de Pinus, vermiculita e perlita da Empresa Eucatex Ltda, que apresentou as seguintes características químicas: $\mathrm{P}$ (resina) $48,1 \mathrm{mg} \mathrm{dm}^{-3}$; M.O. 731,7 $\mathrm{g} \mathrm{dm}^{-3} ; \mathrm{pH}\left(\mathrm{CaCl}_{2}\right)$ 5,2; $\mathrm{K}$ 2,8; Ca 18,8; $\mathrm{Mg}$ 19,5; $\mathrm{H}+\mathrm{Al}$ 27,2; $\mathrm{S} 41,1 ; \mathrm{T}$ 68,3 mmol dm²; V 60,2\%; Cu 0,4; Fe 13,6; Mn 17,1 e Zn $1,4 \mathrm{mg} \mathrm{dm}^{-3}$.

No experimento foi utilizado um esquema fatorial fracionário (1/5) $5^{3}$ com 5 blocos ao acaso (com um total de 25 tratamentos) conforme Conagin \& Jorge (1982). A distribuição utilizada foi: bloco I, tratamentos 111, 254, 342, 435 e 523; no bloco II, 134, 222, 315, 453 e 541; bloco III, 152, 245, 333, 421 e 514; bloco IV, 125, 213, 315, 444 e 532; e bloco $\mathrm{V}, 143,213,324,412$ e 555 (os números dos tratamentos indicam as doses de $\mathrm{N}, \mathrm{P}$ e K, respectivamente).

O P, na forma de superfosfato triplo $\left(78,6 \mathrm{~g} \mathrm{~kg}^{-1}\right.$ $\mathrm{P})$, foi aplicado totalmente no plantio e homogeneizado com o substrato, fornecendo-se as seguintes doses ( $\mathrm{g}$ por planta): 0,$19 ; 0,89 ; 1,59 ; 2,29$; e 2,99. O N e o K foram parcelados durante 40 semanas, em soluções de $\mathrm{NH}_{4} \mathrm{NO}_{3}$ (330 $\mathrm{g} \mathrm{kg}^{-1}$ de $\mathrm{N}$ ) e KCl $\left(497,9 \mathrm{~g} \mathrm{~kg}^{-1}\right.$ de K), respectivamente. As mudas receberam: 1,$25 ; 6,25 ; 11,25$; 16,25 e 21,25 g por planta de $\mathrm{N}$ e 0,$41 ; 3,75 ; 6,22 ; 9,34$ e $12,45 \mathrm{~g}$ por planta de $\mathrm{K}$. Os níveis mais baixos de $\mathrm{P}$ e $\mathrm{K}$ correspondem aos níveis presentes no substrato. Os porta-enxertos receberam $35,7 \%$ das doses de $\mathrm{N}(0,47$; 2,$34 ; 4,22,6,09$ e $7,97 \mathrm{~g}$ por planta) e de $\mathrm{K}(1,41 ; 2,33$; 3,50 e $4,67 \mathrm{~g}$ por planta) o restante sendo fornecido após a enxertia. Foi realizada uma aplicação de $\mathrm{CaCO}_{3}$ e de $\mathrm{CaSO}_{4}$ nas doses de 0,34 e $0,9 \mathrm{~g} \mathrm{dm}^{-3}$ de substrato, respectivamente. Os micronutrientes foram fornecidos através de pulverizações mensais nas seguintes dosagens $\left(\mathrm{g} \mathrm{L}^{-1}\right)$ : B, 0,2; $\mathrm{Mn}, 0,5$ e Zn, 0,6.

A instalação e condução das plantas basearam-se nas recomendações de Carvalho (1998). Os porta-enxertos de limoeiro 'Cravo' (Citrus limonia Osbeck) foram semeados em tubetes de $50 \mathrm{~cm}^{3}$ e transplantados aos 4 meses para vasos com capacidade para 3,8L, quando foram iniciados os tratamentos. Após 4 meses de cultivo, realizou-se a enxertia em "T" invertido com borbulhas de laranjeira 'Valência' (Citrus sinnensis L. Osbeck), e o amarrio foi realizado com fitilho plástico. Aos 20 dias após a enxertia foi realizado 0 forçamento da brotação da borbulha através da decapitação do porta-enxerto acima da enxertia, por ocasião da retirada do fitilho. As mudas foram conduzidas até o ponto de muda de haste única, 6 meses após a enxertia.

Avaliaram-se a produção de material seco das folhas, raízes, caule, parte aérea e total (g por planta); área foliar $\left(\mathrm{cm}^{2}\right)$; relação raízes/parte aérea; altura de plantas $(\mathrm{cm})$; volume radicular $\left(\mathrm{cm}^{3}\right)$; relação área foliar/material seco das folhas e total $\left(\mathrm{cm}^{2} \mathrm{~g}^{-1}\right)$ dos porta enxertos e das mudas; diâmetro do caule na altura de enxertia $(\mathrm{mm})$ para o porta-enxerto e comprimento dos surtos vegetativos de crescimento $(\mathrm{cm})$ das mudas. 
A análise de variância foi realizada para todos os parâmetros avaliados, em função das doses dos nutrientes. Foram ajustadas funções de resposta do tipo $\mathrm{Y}=\gamma_{0}+\gamma_{1} \mathrm{~N}+$ $\gamma_{2} \mathrm{~N}^{2}+\gamma_{3} \mathrm{P}+\gamma_{4} \mathrm{P}^{2}+\gamma_{5} \mathrm{~K}+\gamma_{6} \mathrm{~K}^{2}+\gamma_{7} \mathrm{NP}+\gamma_{8} \mathrm{NK}+\gamma_{9} \mathrm{PK}$, onde $\mathrm{Y}$ é a variável dependente, $\gamma$ os coeficientes da regressão e $\mathrm{N}$, $\mathrm{P}$ e $\mathrm{K}$ as doses utilizadas dos nutrientes (TABELAS 1 e 2). As análises estatísticas foram realizadas utilizando 0 programa estatístico SAS (SAS Institute, 1996). Quando as respostas foram significativas apenas para 1 ou 2 nutrientes, as funções foram simplificadas, considerando-se os níveis menores dos demais nutrientes. Determinaram-se as doses dos nutrientes que proporcionaram as maiores produções através do cálculo $d x / d y=0$, seguindo os procedimentos de Cantarella et al. (1992). Estudaram-se as correlações (r) entre os parâmetros avaliados.

\section{RESULTADOS E DISCUSSÃO}

$\mathrm{Na}$ TABELA 3 estão as equações obtidas para as relações entre adubação NPK e os paramêtros avaliados.
Os valores máximos da produção de material seco do caule, parte aérea e total do porta-enxerto limoeiro 'Cravo' foram 19,01; 31,07 e 45,32 g por planta, nas doses de 2,67; 2,66; e 2,29 g por planta de K. Na África do Sul, Miller et al. (1993), trabalhando com 5 porta-enxertos de citros também obtiveram aumentos na produção do material seco total das plantas com o fornecimento de $\mathrm{K}$ na solução nutritiva até uma dose intermediária (150 $\left.\mathrm{mg} \mathrm{L}^{-1}\right)$ entretanto, quando elevaram a dose para $300 \mathrm{mg} \mathrm{L}^{-1}$ não houve resposta.

$\mathrm{Na}$ produção de folhas houve interação entre $\mathrm{N}$ e K. A produção máxima de material seco de folhas $(12,95 \mathrm{~g}$ por planta) foi obtida com as doses de 3,96 e 2,66 g por planta, de $\mathrm{N}$ e de $\mathrm{K}$, respectivamente. Essa interação nas folhas pode ser explicada analisando-se o trabalho de Moorby \& Besford (1983), que mostraram existir relações positivas entre crescimento das folhas, fotossíntese e fornecimento de $\mathrm{K}$. Além disso, como o $\mathrm{N}$ é constituinte de todas as proteínas, atua diretamente sobre o crescimento e metabolismo vegetal.

$\mathrm{O}$ aumento nas doses de $\mathrm{N}$ interferiu negativamente na produção de material seco das raízes em ambas as

TABELA 1 - Superfícies de resposta obtidas para as variáveis estudadas em função das doses de N, P e K para o limoeiro 'Cravo'.

\begin{tabular}{|c|c|c|c|c|c|c|c|c|c|c|c|}
\hline Variável & $g_{0}$ & $\mathrm{~N}$ & $\mathrm{~N}^{2}$ & $P$ & $\mathrm{P}^{2}$ & K & $\mathrm{K}^{2}$ & NP & NK & PK & Teste $\mathrm{F}^{(1)}$ \\
\hline MS raízes & 15,233 & $-1,123$ & 0,028 & $-0,173$ & $-0,017$ & 0,0163 & $-0,146$ & $-0,0057$ & 0,05 & 0,057 & $\mathrm{~N}_{\mathrm{L}}{ }^{* *}$ \\
\hline MS caule & 16,263 & $-0,453$ & $-0,056$ & $-0,484$ & $-0,076$ & 2,3 & $-0,431$ & 0,086 & 0,05 & $-0,137$ & $\mathrm{~K}_{\mathrm{L}}{ }^{*} \mathrm{~K}_{\mathrm{Q}}{ }^{* *}$ \\
\hline MS folhas & 10,042 & 0,483 & $-0,073$ & 0,531 & $-0,409$ & 1,347 & $-0,27$ & 0,146 & 0,022 & 0,071 & $\mathrm{~K}_{\mathrm{LQ}}{ }^{*} \mathrm{~N}_{\mathrm{Q}}{ }^{*}$ \\
\hline MS p.aérea & 26,753 & $-0,08$ & $-0,11$ & $-0,43$ & $-0,318$ & 3,329 & $-0,629$ & 0,213 & 0,06 & $-0,056$ & $\mathrm{~K}_{\mathrm{Q}}^{*}$ \\
\hline MS total & 41,985 & $-1,202$ & $-0,082$ & $-0,603$ & $-0,335$ & 3,492 & $-0,775$ & 0,156 & 0,11 & 0,0008 & $\mathrm{~K}_{\mathrm{L}}{ }^{*} \mathrm{~K}_{\mathrm{Q}}{ }^{* *}$ \\
\hline Diâm caule & 87,795 & $-1,174$ & $-0,115$ & 3,743 & $-1,027$ & 6,842 & $-1,066$ & 0,042 & $-0,223$ & $-0,854$ & $\mathrm{~K}_{\mathrm{LQ}}{ }^{* *}$ \\
\hline $\mathrm{H}$ plantas & 112,52 & $-0,323$ & $-0,283$ & 0,189 & $-0,889$ & 10,514 & $-1,619$ & 0,89 & $-0,147$ & $-0,526$ & $\mathrm{~K}_{\mathrm{LQ}}$ ** \\
\hline Vol raízes & 37,986 & $-1,339$ & $-0,105$ & 7,245 & $-1,224$ & 2,563 & $-0,668$ & $-0,457$ & 0,171 & $-0,025$ & $\mathrm{P}_{\mathrm{L}}^{*}$ \\
\hline Raíz/p.aérea & 0,552 & $-0,036$ & 0,002 & 0,018 & $-0,0008$ & $-0,032$ & 0,002 & $-0,0057$ & 0,0009 & 0,002 & $\mathrm{~N}_{\mathrm{L}}{ }^{*}$ \\
\hline Área foliar & 1192,7 & 46,708 & $-9,826$ & 165,58 & $-52,492$ & 153,21 & $-27,909$ & 5,139 & 9,692 & $-11,244$ & $\mathrm{~K}_{\mathrm{L}}^{*} \mathrm{~N}_{\mathrm{Q}}{ }^{* *}$ \\
\hline $\begin{array}{l}\text { Área foliar/ } \\
\text { MS total }\end{array}$ & 30,34 & 1,833 & $-0,161$ & 4,409 & $-1,096$ & $-0,189$ & 0,038 & 0,107 & 0,293 & $-0,149$ & n.s. \\
\hline
\end{tabular}

(1) $L$ e $Q$ indicam efeitos significativos lineares e quadráticos, respectivamente.

TABELA 2 - Superfícies de resposta obtidas para as variáveis estudadas em função das doses de N, P e K para a laranjeira 'Valência'/'Cravo'.

\begin{tabular}{|c|c|c|c|c|c|c|c|c|c|c|c|}
\hline Variável & $\gamma_{0}$ & $\mathrm{~N}$ & $\mathrm{~N}^{2}$ & $\mathrm{P}$ & $\mathrm{P}^{2}$ & $\mathrm{~K}$ & $\mathrm{~K}^{2}$ & NP & NK & $\mathrm{PK}$ & Teste $\mathrm{F}^{(1)}$ \\
\hline MS raízes & 26,534 & $-1,285$ & 0,023 & $-0,075$ & 0,142 & 0,011 & $-0,039$ & $-0,094$ & 0,032 & 0,08 & $\mathrm{~N}_{\mathrm{L}}{ }^{* * *} \mathrm{~N}_{\mathrm{Q}}{ }^{*}$ \\
\hline MS caule & 15,045 & $-0,53$ & $-0,0006$ & $-0,805$ & 0,205 & 0,465 & $-0,044$ & $-0,026$ & 0,009 & 0,054 & $\mathrm{~N}_{\mathrm{L}}{ }^{*}$ \\
\hline MS folhas & 13,015 & 0,692 & $-0,05$ & $-1,437$ & 0,269 & 0,484 & $-0,049$ & $-0,042$ & $-0,006$ & 0,195 & $\mathrm{~N}_{\mathrm{L}}{ }^{* *} \mathrm{~N}_{\mathrm{Q}}{ }^{* \star *}$ \\
\hline MS p. aérea & 28,06 & 0,162 & $-0,051$ & $-2,242$ & 0,474 & 0,949 & $-0,092$ & $-0,068$ & 0,003 & 0,249 & $\mathrm{~N}_{\mathrm{Q}}{ }^{* \star \star} \mathrm{K}_{\mathrm{Q}}{ }^{*}$ \\
\hline MS total & 54,594 & $-1,123$ & $-0,027$ & $-2,317$ & 0,616 & 0,96 & $-0,131$ & $-0,162$ & 0,035 & 0,329 & $\mathrm{~N}_{\mathrm{L}}{ }^{*} \mathrm{~K}_{\mathrm{Q}}{ }^{*}$ \\
\hline Surto veg. 1 & 24,169 & $-1,155$ & 0,033 & $-1,188$ & 0,623 & 0,127 & $-0,026$ & $-0,094$ & $-0,0001$ & 0,037 & $\mathrm{~N}_{\mathrm{L}}{ }^{* * *} \mathrm{~N}_{\mathrm{Q}}{ }^{* *}$ \\
\hline Surto veg. 2 & 26,392 & 1,741 & $-0,103$ & $-2,092$ & 0,742 & $-0,981$ & 0,029 & $-0,252$ & 0,026 & 0,486 & $\mathrm{~N}_{\mathrm{LQ}}{ }^{* * *} \mathrm{~N}_{\mathrm{P}}{ }^{*}$ \\
\hline $\mathrm{H}$ plantas & 50,562 & 0,586 & $-0,07$ & $-3,28$ & 1,365 & $-0,854$ & 0,003 & $-0,346$ & 0,024 & 0,523 & $N_{Q}^{* \star *} N_{P}^{* *}$ \\
\hline Vol. Raízes & 57,227 & $-3,652$ & 0,076 & $-2,552$ & 0,683 & 1,348 & $-0,114$ & $-0,001$ & 0,036 & $-0,181$ & $\mathrm{~N}_{\mathrm{LQ}}{ }^{* \star *}$ \\
\hline Raiz/p.aérea & 0,959 & $-0,739$ & 0,004 & 0,113 & $-0,031$ & $-0,01$ & 0,0005 & 0,0007 & $-0,0002$ & $-0,004$ & $\mathrm{~N}_{\mathrm{LQ}}{ }^{* * *}$ \\
\hline Área foliar & 1385,3 & 135,68 & $-8,577$ & $-168,41$ & 45,015 & 131,806 & $-9,403$ & $-5,681$ & $-2,562$ & 25,357 & $\mathrm{~N}_{\mathrm{LQ}}{ }^{* * *} \mathrm{~K}_{\mathrm{LQ}}{ }^{*}$ \\
\hline $\begin{array}{l}\text { Área foliar/ } \\
\text { MS total }\end{array}$ & 22,0 & 4,607 & $-0,223$ & 0,372 & 0,37 & 2,039 & $-0,113$ & $-0,0875$ & $-0,025$ & 0,065 & $\mathrm{~N}_{\mathrm{LQ}}{ }^{* * *} \mathrm{~K}_{\mathrm{LQ}}{ }^{*}$ \\
\hline
\end{tabular}

(1) $L$ e $Q$ indicam efeitos significativos lineares e quadráticos, respectivamente. 
avaliações.Pode-se observar que houve decréscimos de 14,7 a 8,2 g para o limoeiro 'Cravo' e de 24,9 a 9,2 g para a laranjeira 'Valência'/'Cravo', das doses menores (0,47 e 1,25 $\mathrm{g}$ por planta de $\mathrm{N})$ para as maiores $(7,97$ e 21,25 g por planta de N). Esses resultados estão de acordo com os obtidos por Ford et al. (1957) e Smith (1965), que também observaram reduções no sistema radicular de plantas cítricas, com a utilização de doses elevadas de $\mathrm{N}$. Isso pode ser considerado um mecanismo de adaptação das plantas para aumentar o volume de solo ou substrato explorado pelas raízes.

A produção de material seco das folhas da laranjeira 'Valência' variou de forma quadrática ao fornecimento de $\mathrm{N}$. $\mathrm{Na}$ dose de 1,25 g por planta a produção foi de $13,63 \mathrm{~g} \mathrm{e} \mathrm{a}$ produção máxima de $14,77 \mathrm{~g}$ foi obtida com a dose de 6,02 $g$ por planta. Na produção de material seco da parte aérea e total das mudas houve interação entre $\mathrm{N}$ e K, e as produções máximas (30,76 e 56,42 g por planta) foram obtidas com 1,62 e 2,47 g por planta de $\mathrm{Ne} \mathrm{5,44} \mathrm{e} \mathrm{4,23} \mathrm{g} \mathrm{por} \mathrm{planta} \mathrm{de} \mathrm{K.}$

O diâmetro do caule é a característica morfológica do porta-enxerto que determina a possibilidade de realização da enxertia. Este parâmetro e a altura de plantas correlacionaram-se direta e significativamente, com um $r=0,65^{\text {t**. }}$. Os resultados obtidos para o limoeiro 'Cravo', mostram efeito quadrático das doses de $\mathrm{K}$ sobre a altura e diâmetro do caule. Houve variações no diâmetro de 99,21 a $95,35 \mathrm{~mm}$, obtidos com as doses de $\mathrm{K}$ mais baixa e mais alta (0,42 e 4,67 g por planta, respectivamente), e o maior diâmetro $(98,03 \mathrm{~mm})$ foi obtido com a dose de 3,08 $\mathrm{g}$ por planta. Os resultados de altura de plantas variaram de 116,38 a 125,32 cm, com a altura máxima $(128,84 \mathrm{~cm})$ obtida com a dose de $3,19 \mathrm{~g}$ por planta.

Após a enxertia, uma causa freqüente do atraso na formação da muda cítrica é a interrupção do surto vegetativo da borbulha. Para a formação da muda, o enxerto pode necessitar de até 3 surtos de crescimento, entretanto, o ideal é que a muda esteja pronta com um único surto, uma vez que cada interrupção no crescimento pode atrasar a formação da muda. Araújo \& Souza (1994) observaram que mudas formadas com 2 surtos de crescimento atingiram a altura de desponte $(60 \mathrm{~cm}) 2,5$ meses mais tarde que as mudas formadas em um único surto.

Neste estudo, foram gastos 14 meses para a produção da muda desde a semente; e o crescimento da brotação da borbulha ocorreu em 2 surtos vegetativos, provavelmente devido às altas temperaturas observadas após a enxertia, realizada no mês de janeiro. De acordo com

TABELA 3 - Função de resposta ajustada para os termos significativos obtidos para o limoeiro 'Cravo' e laranjeira 'Valência'/ 'Cravo'. As condições indicam os níveis dos nutrientes não significativos para os quais as equações foram ajustadas.

\begin{tabular}{|c|c|c|c|}
\hline Variável & Equação & Condição (g por planta) & $\mathrm{R}^{2}$ \\
\hline \multicolumn{4}{|c|}{ Limoeiro 'Cravo' } \\
\hline MSTotal & $Y=-0,775 K^{2}+3,544 K+41,269$ & $P=0,19$ e $N=0,47$ & 0,18 \\
\hline MSP.aérea & $Y=-0,626 K^{2}+3,346 K+26,617$ & $P=0,19$ e $N=0,47$ & 0,22 \\
\hline MScaule & $Y=-0,431 K^{2}+2,298 K+15,951$ & $P=0,19$ e $N=0,47$ & 0,42 \\
\hline MSFolhas & $Y=-0,073 N^{2}+0,511 N-0,27 K^{2}+1,36 K+0,022 N K+10,128$ & $P=0,19$ & 0,18 \\
\hline MSRaízes & $Y=0,028 N^{2}-1,104 N+15,186$ & $P=0,19$ e $K=0,42$ & 0,60 \\
\hline Altura & $Y=-1,619 K^{2}+10,345 K+112,318$ & $P=0,19$ e $N=0,47$ & 0,27 \\
\hline Diam & $Y=-0,107 K^{2}+0,658 K+8,79$ & $P=0,19$ e $N=0,47$ & 0,60 \\
\hline AF & $Y=-9,826 N^{2}+47,68 N-27,91 K^{2}+151,1 K+9,692 N K+1222,3$ & $P=0,19$ & 0,27 \\
\hline $\mathrm{Rz} / \mathrm{PA}$ & $Y=0,002 N^{2}-0,037 N+0,556$ & $P=0,19$ e $K=0,42$ & 0,47 \\
\hline Volraízes & $Y=-1,224 P^{2}+7,02 P+38,986$ & $\mathrm{~N}=0,47$ e $\mathrm{K}=0,42$ & 0,51 \\
\hline \multicolumn{4}{|c|}{ 'Valência'/ 'Cravo' } \\
\hline MSTotal & $Y=-0,027 N^{2}-0,016 N-0,131 K^{2}+1,023 K+0,035 N K+54,271$ & $P=0,19$ & 0,47 \\
\hline MSP.aérea & $Y=-0,051 N^{2}+0,149 N-0,092 K^{2}+0,996 K+0,003 N K+27,651$ & $P=0,19$ & 0,74 \\
\hline MScaule & $Y=-0,0006 N^{2}-0,539 N+15,076$ & $P=0,19$ e $K=0,42$ & 0,69 \\
\hline MSFolhas & $Y=-0,05 N^{2}+0,692 N+12,957$ & $P=0,19$ e $K=0,42$ & 0,69 \\
\hline MSRaízes & $Y=0,023 N^{2}-1,303 N+26,529$ & $P=0,19$ e $K=0,42$ & 0,69 \\
\hline Surto 1 & $Y=0,033 N^{2}-1,173 N+24,016$ & $P=0,19$ e $K=0,42$ & 0,61 \\
\hline Surto 2 & $Y=-0,103 N^{2}+1,94 N+0,742 P^{2}-1,893 P-0,252 N P+25,995$ & $K=0,42$ & 0,60 \\
\hline Altura & $Y=-0,07 N^{2}+0,596 N+1,365 P^{2}-3,066 P-0,346 N P+50,217$ & $\mathrm{~K}=0,42$ & 0,75 \\
\hline AF & $Y=-8,58 N^{2}+134,6 N-9,40 K^{2}+136,6 K-2,56 N K+1354,9$ & $P=0,19$ & 0,71 \\
\hline $\mathrm{Rz} / \mathrm{PA}$ & $Y=0,004 N^{2}-0,74 N+0,975$ & $P=0,19$ e $K=0,42$ & 0,44 \\
\hline Volraízes & $Y=0,076 N^{2}-3,63 N+57,286$ & $P=0,19$ e $K=0,42$ & 0,79 \\
\hline AF/MStotal & $Y=-0,223 N^{2}+4,59 N-0,113 K^{2}+2,051 K-0,025 N K+22,084$ & $P=0,19$ & 0,59 \\
\hline
\end{tabular}

$\mathrm{MS}$ = material seco; $\mathrm{AF}$ = área foliar; Rz/PA = relação raízes / parte aérea; $\mathrm{Vol}_{\text {raízes }}=$ volume radicular; $\mathrm{AF} / \mathrm{MS}_{\text {total }}=$ razão de área foliar. 
Carvalho (1998), este fator climático pode paralisar o crescimento e reduzir o pegamento das borbulhas. Quando as plantas iniciaram o primeiro surto vegetativo, observouse que o nitrogênio influenciou negativamente, sendo que 0 maior crescimento $(22,6 \mathrm{~cm})$ foi obtido com a menor dose de $\mathrm{N}(1,25 \mathrm{~g}$ por planta). Na dose mais elevada $(21,25 \mathrm{~g}$ por planta) o comprimento do surto foi $13,99 \mathrm{~cm}$. Já no segundo, obteve-se interação entre $\mathrm{Ne} \mathrm{P}$, observando-se crescimento maior nas doses de 4,24 e 1,54 g por planta de $\mathrm{N}$ e de $\mathrm{P}$, respectivamente. Graff et al. (1994), em viveiro de campo, não observaram efeitos das pulverizações foliares com N, Mg, $\mathrm{S}, \mathrm{Zn}, \mathrm{Mn}$ e B sobre o comprimento e o diâmetro das brotações de laranjeiras 'Valência' e 'Pera' enxertadas em limoeiro 'Cravo', porém o ponto de desponte foi atingido em um único surto de crescimento.

Analisando-se a altura final das mudas observase também, a interação entre $\mathrm{N}$ e $\mathrm{P}$, sendo que as plantas mais altas $(52,6 \mathrm{~cm})$ foram obtidas com as doses de 1,25 e 2,99 g por planta de $\mathrm{Ne} \mathrm{P}$, respectivamente. O segundo crescimento foi mais intenso que o primeiro e relacionouse mais com a altura final das plantas $\left(r=0,90^{* * * *}\right.$ e $0,71^{* * *}$, respectivamente).

A determinação da área foliar é importante, porque as folhas são as principais responsáveis pela captação da energia solar e pela produção de material orgânico através da fotossíntese. Os resultados obtidos mostraram que houve interação entre os nutrientes $\mathrm{N} \mathrm{e}$ $\mathrm{K}$ na área foliar, tanto no porta-enxerto, quanto na muda. As áreas foliares máximas $\left(1578,8\right.$ e $\left.2251,4 \mathrm{~cm}^{2}\right)$ foram obtidas com 4,11 e 6,90 g por planta de $\mathrm{N} \mathrm{e} \mathrm{3,42} \mathrm{e} \mathrm{6,32} \mathrm{g}$ por planta de K, para o limoeiro 'Cravo' e a laranjeira 'Valência', respectivamente. Maust \& Williamson (1994) trabalhando com mudas observaram as maiores áreas foliares nos níveis intermediários de $\mathrm{N}$.

A relação raízes/parte aérea é útil para estudar-se 0 equilíbrio entre os órgãos, devido às modificações no ambiente, uma vez que indica a existência de uma interdependência entre os órgãos no balanço por água, nutrientes e carbono. Marschner (1995) e Witt (1997) afirmaram que o N interfere diretamente nesse balanço, alterando a morfologia da planta. Quando há baixa disponibilidade do nutriente, há menor crescimento da parte aérea e as raízes são longas e sem divisões. Nos níveis intermediários, há desenvolvimento e divisão adequados do sistema radicular. No excesso do nutriente, observa-se excesso de divisão das raízes, porém o sistema radicular é reduzido e há estímulo para 0 desenvolvimento da parte aérea.

O papel do $\mathrm{N}$ no balanço entre o sistema radicular e a parte aérea da muda também pode ser evidenciado, analisando-se a relação raízes/parte aérea. O $\mathrm{N}$ atuou negativamente sobre a relação, sendo que a relação máxima $(0,54)$ foi obtida na menor dose de $\mathrm{N}(0,47 \mathrm{~g}$ por planta). Aumentando-se a dose até $7,97 \mathrm{~g}$ ocorreu redução na relação para 0,39 . Após a enxertia, o $\mathrm{N}$ continuou a interferir nessa relação, porém houve efeito quadrático das doses do nutriente e, o ponto onde obteve-se a relação mínima $(0,63)$ foi com a dose de 9,25 g. Nas doses mínima e máxima $(1,25$ e 21,25 g) a relação variou de 0,89 até 1,21 .

Alterações na morfologia do sistema radicular, como no seu volume, podem alterar a eficiência de absorção de água e nutrientes no solo, principalmente aqueles mais imóveis no solo, e cujo contato com as raízes ocorre por difusão. As mudas com um sistema radicular mais desenvolvido, quando levadas ao campo, provavelmente terão melhores condições de estabelecimento. O fósforo proporcionou aumentos no volume radicular do porta-enxerto limoeiro 'Cravo'. O volume das raízes variou de 40,28 a $49,05 \mathrm{~cm}^{3}$, sendo que o maior volume foi obtido com a dose de $2,86 \mathrm{~g}$ por planta de P. Essa pode ser considerada a dose adequada para a produção de mudas nesse sistema, pois estimulou 0 estabelecimento do sistema radicular da futura muda. $O$ papel do $\mathrm{P}$ no aumento do volume de raízes pode estar no aumento do número e do comprimento das radicelas (Marschner, 1995). Nos viveiros da África do Sul são utilizados entre 0,32 e $0,42 \mathrm{~g} \mathrm{dm}^{-3}$ de $P$ como superfosfato simples (Coetzee et al., 1993). Estes são valores inferiores aos da resposta obtida neste trabalho $\left(0,75 \mathrm{~g} \mathrm{dm}^{-3}\right)$, porém deve ser considerado que estas doses são aplicadas no transplantio e posteriormente as plantas recebem adubação NPK complementar durante o desenvolvimento.

De acordo com Castle (1978), após a enxertia há favorecimento do crescimento da parte aérea da muda em detrimento do sistema radicular. Esse fato pode ser determinado através da observação da variação do volume radicular, pois após a enxertia houve grande redução no sistema radicular com o aumento das doses de N. Podese comprovar que até a enxertia, o menor volume de raízes foi $40,8 \mathrm{~cm}^{3}$, após este período, houve reduções até para $14,32 \mathrm{~cm}^{3}$, na maior dose de $\mathrm{N}(21,25 \mathrm{~g}$ por planta). $\mathrm{Na}$ menor dose de $\mathrm{N}$ (1,25 g por planta) o volume foi de $52,86 \mathrm{~cm}^{3}$, indicando que nessa dose, ainda houve pequeno crescimento de raízes, pois o maior vo-lume na época de enxertia foi de $48,05 \mathrm{~cm}^{3}$.

O quociente entre a área foliar e a produção de material seco total das plantas é chamado de razão de área foliar, e fornece uma medida da dimensão relativa do aparelho assimilador das plantas (Magalhães, 1985). Houve interação entre $\mathrm{N}$ e $\mathrm{K}$ sobre este fator, e a maior relação $\left(52,87 \mathrm{~cm}^{2} \mathrm{~g}^{-1}\right)$ foi obtida nas doses de 9,85 e 7,99 $\mathrm{g}$ de $\mathrm{N}$ e de $\mathrm{K}$, respectivamente.

As altas correlações obtidas entre as variáveis mostram que as medidas utilizadas foram adequadas para a avaliação do crescimento das plantas. Assim, as correlações obtidas entre diâmetro do caule do limoeiro 'Cravo' e a produção de material seco total $\left(0,683^{* * *}\right)$; produção das raízes $\left(0,688^{*+*}\right)$ e o volume radicular $\left(0,649^{*+*}\right)$, indicam que existe interdependência positiva entre essas variáveis.

Para as mudas de laranjeira 'Valência'/'Cravo', as correlações significativas e positivas existentes entre a razão de área foliar e a produção de material seco total $\left(0,631^{* *}\right)$ e altura das plantas $\left(0,706^{* \prime \prime}\right)$, indicam que este também é um parâmetro adequado para a avaliação das mudas. 
Deste modo, as doses de melhor resposta para a produção das mudas, considerando-se a razão de área foliar foram 9,85 e 7,99 g por planta de $\mathrm{N}$ e de K. No entanto, considerando-se a produção total de material seco foram 2,47 e 4,23 g por planta de $\mathrm{N}$ e de $\mathrm{K}$, respectivamente. Considerando-se a área foliar as doses de nutrientes são sempre maiores, mostrando o efeito sobre a expansão das folhas. Os resultados mostram que este aumento de área foliar não reflete-se em aumentos de material seco. No entanto, considerando-se a quantidade de fertilizantes que foi aplicada nos porta-enxertos, de $37,5 \%$ do total de $\mathrm{N}$ e de $\mathrm{K}$, obtém-se os valores de 3,69 e 3,00 g por planta, respectivamente. Esses valores são muito próximos dos obtidos.

$\mathrm{O}$ volume das raízes do porta-enxerto teve altas correlações com o material seco total e das raízes do porta-enxerto $\left(0,727^{\text {t*t }}\right.$ e $\left.0,808^{* * *}\right)$ e também com o material seco total das mudas $\left(0,773^{+* *+}\right)$.

\section{CONCLUSÕES}

As doses de fertilizantes que levam às melhores respostas para produção de mudas de laranjeira 'Valência'/ 'Cravo' sobre o porta-enxertos de limoeiro 'Cravo' são: N, 9,85; P, 2,86; K, 7,99 g por planta, sendo que o $\mathrm{N}$ e o K são fornecidos $37,5 \%$ da dose ao porta-enxerto e o restante à muda, e o P é aplicado totalmente no plantio.

\section{AGRADECIMENTO}

\section{À FAPESP, pelo financiamento da pesquisa.}

\section{REFERÊNCIAS BIBLIOGRÁFICAS}

ARAÚJO, S.C.; SOUZA, M. Influência da adubação e vigor dos ramos porta borbulhas na qualidade dos enxertos de citros. Ciência e Prática, v.18, p.151-157, 1994.

BEVINGTON, K.; CASTLE, W.S. Annual root growth pattern of young citrus trees in relation to shoot growth, soil temperature, and soil water content. Journal of the American Society for Horticultural Science, v.110, p.840-845, 1985.

CANTARELLA, H.; QUAGGIO, J.A.; BATAGLIA, O.C.; RAIJ, B. van Response of citrus to NPK fertilization in a network of field trials in São Paulo State, Brazil. In: INTERNATIONAL CITRUS CONGRESS, Acireale, 1992. Proceedings. Acireale: International Society of Citriculture, 1992. p.607-612.

CARVALHO, S.A. Produção de porta-enxertos cítricos, sob doses crescentes de nitrato de potássio. Pesquisa Agropecuária Brasileira, v.29, p.87-90, 1994.

CARVALHO, S.A. Estratégias para estabelecimento e manutenção de matrizes, borbulheiras e viveiro de citros em ambiente protegido. In: SEMINÁRIO INTERNACIONAL DE CITROS-TRATOS CULTURAIS, 5., Bebedouro, 1998. Anais. Bebedouro: Fundação Cargill, 1998. p.67-101.

CARVALHO, S.A.; SOUZA, M. de. Resposta do limoeiro 'Cravo' em sementeira a dois métodos de aplicação de superfosfato simples. In: CONGRESSO BRASILEIRO DE FRUTICULTURA, 9., Campinas, 1988. Anais. Campinas: Sociedade Brasileira de Fruticultura, 1988. v.2, p.429-434.

CARVALHO, S.A.; LARANJEIRA, F.F. Protótipo de viveiro de mudas certificadas e borbulheiras sob telado à prova de afídeos do Centro de Citricultura-IAC. Laranja, v.15, p.213-220, 1994.
CASTLE, W.S. Citrus root systems: their structure, function, growth, and relationship to tree performance. In: INTERNATIONAL CITRUS CONGRESS, Sidney, 1978. Proceedings. Sidney: International Society of Citriculture, 1978. p.62-69.

CASTLE, W.S.; FERGUNSON, J.J. Current status of greenhouse and container production of citrus nursery trees in Florida. Proceedings of the Florida State Horticultural Society, v.95, p.42-46, 1982.

COETZEE, J.G.K.; ESSELEN, L.; VAN ROOYEN, A. Fertilization of nursery trees - alternative method. In: WORLD CONGRESS OF THE INTERNATIONAL SOCIETY OF CITRUS NURSERYMEN, 2., 1993 Pietermaritzburg. Proceedings. Pietermaritzburg: International Society of Citrus Nurserymen, 1993. p.143-150.

CONAGIN, A.; JORGE, J.P.N. Delineamento (1/5) $(5 \times 5 \times 5)$ em blocos. Bragantia, v.41, p.155-168, 1982.

DECARLOS NETO, A.; DE GRANDI, A.J.; VICHIATO, M.; AMARAL, AM. do; SOUZA, M. de. Viabilização do uso de tubetes para obter o porta-enxerto limoeiro 'Cravo' com "solução de arranque". In: CONGRESSO BRASILEIRO DE FRUTICULTURA, 13., Salvador, 1994. Anais. Salvador: Sociedade Brasileira de Fruticultura, 1994. v.3, p.400-401.

FORD, H.W.; REUTHER, W.; SMITH, P.F. Effect of nitrogen on root development of Valencia orange trees. Proceedings of the Florida State Horticultural Society, v.70, p.234-244, 1957.

GRAFF, C.C.D.; MARINHO, C.S.; SOUTO, R.F.; PAIVA, L.V.; SOUZA SOBRINHO, F. Efeito da fertilização foliar após a enxertia no crescimento e nutrição da muda da laranjeira (Citrus sinensis (L.) Osbeck cv. Valência). Ciência e Prática, v.18, p.264-267, 1994.

LEGAZ, F.; SERNA, M.D.; PRIMO-MILLO, E. Mobilization of the reserve N in citrus. Plant and Soil, v.173, p.205-210, 1995.

MAGALHÃES, A.C.N. Análise quantitativa do crescimento. In: FERRI, M.G. (Coord.) Fisiologia vegetal. 2.ed. São Paulo: Ed. Pedagógica e Universitária, 1985. v.1, p.333-350.

MARSCHNER, $\mathrm{H}$. Mineral nutrition of higher plants New York: Academic Press, 1995. 889p.

MAUST, B.E.; WILLIAMSON, J.G. Nitrogen nutrition of containerized citrus nursery plants. Journal of the American Society for Horticultural Science, v.119, p.195-201, 1994.

MILLER, J.E.; HOFMAN, P.J.; BERRY, R.K. Physiological and nutritional responses of five citrus rootstocks to potassium. Journal of the Southern African Society for Horticultural Sciences, v.3, p.20-23, 1993.

MOORBY, J.; BESFORD, R.T. Mineral nutrition and growth. In: GÖTTING, A.P.; HARVARD, M.H. Inorganic plant nutrition. Berlin: Springer-Verlag, 1983. v.15B, p.481-527.

RESENDE, L.P.; AMARAL, A.M.; CARVALHO, S.A.; SOUZA, M. Volume do substrato e superfosfato simples na formação do limoeiro 'Cravo' em vasos: I. Efeitos no crescimento vegetativo. Laranja, v.16, p.155-164, 1995.

SAS INSTITUTE. The SAS-sytstem for windows: release 6.11 (software). Cary: Statistical Analysis System Institute, 1996.

SMITH, P.F. Effect of nitrogen source and placement on the root development of Valencia oranges trees. Proceedings of the Florida State Horticultural Society, v.78, p.55-59, 1965.

SYVERTSEN, J.P.; LLOYD, J. Citrus. In: SCHAEFFER, B.; ANDERSEN, P. (ed.). Handbook of environmental physiology of fruits crops: subtropical and tropical crops. Boca Raton: CRC Press, 1994. v.2, p.65-99.

WITT, H.H. Root growth of trees as influenced by physical and chemical soilfactors. Acta Horticulturae, n.450, p.205-214, 1997.

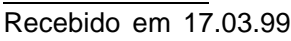

